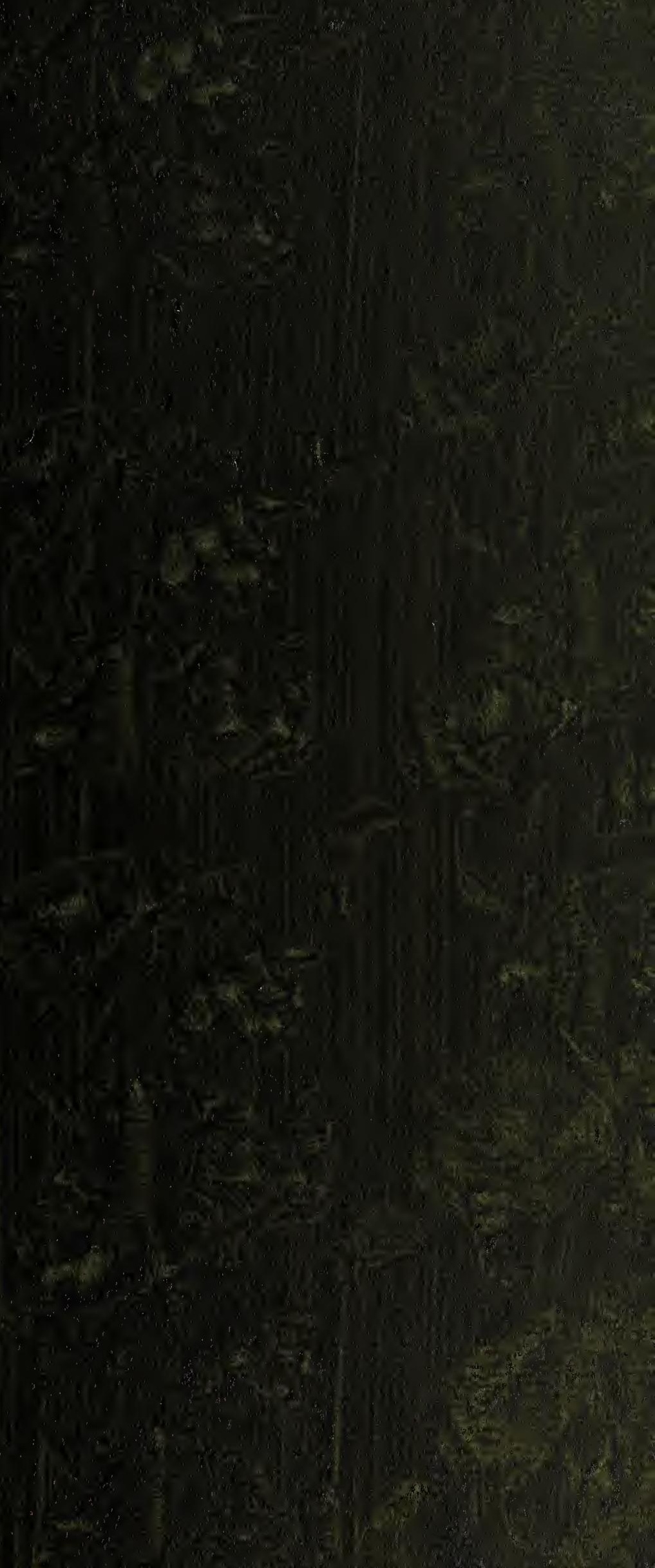


C.S.RAFINESQUE

Zoologieal rbotanical papers -

Also" the fleasures and duties of ruealct - 




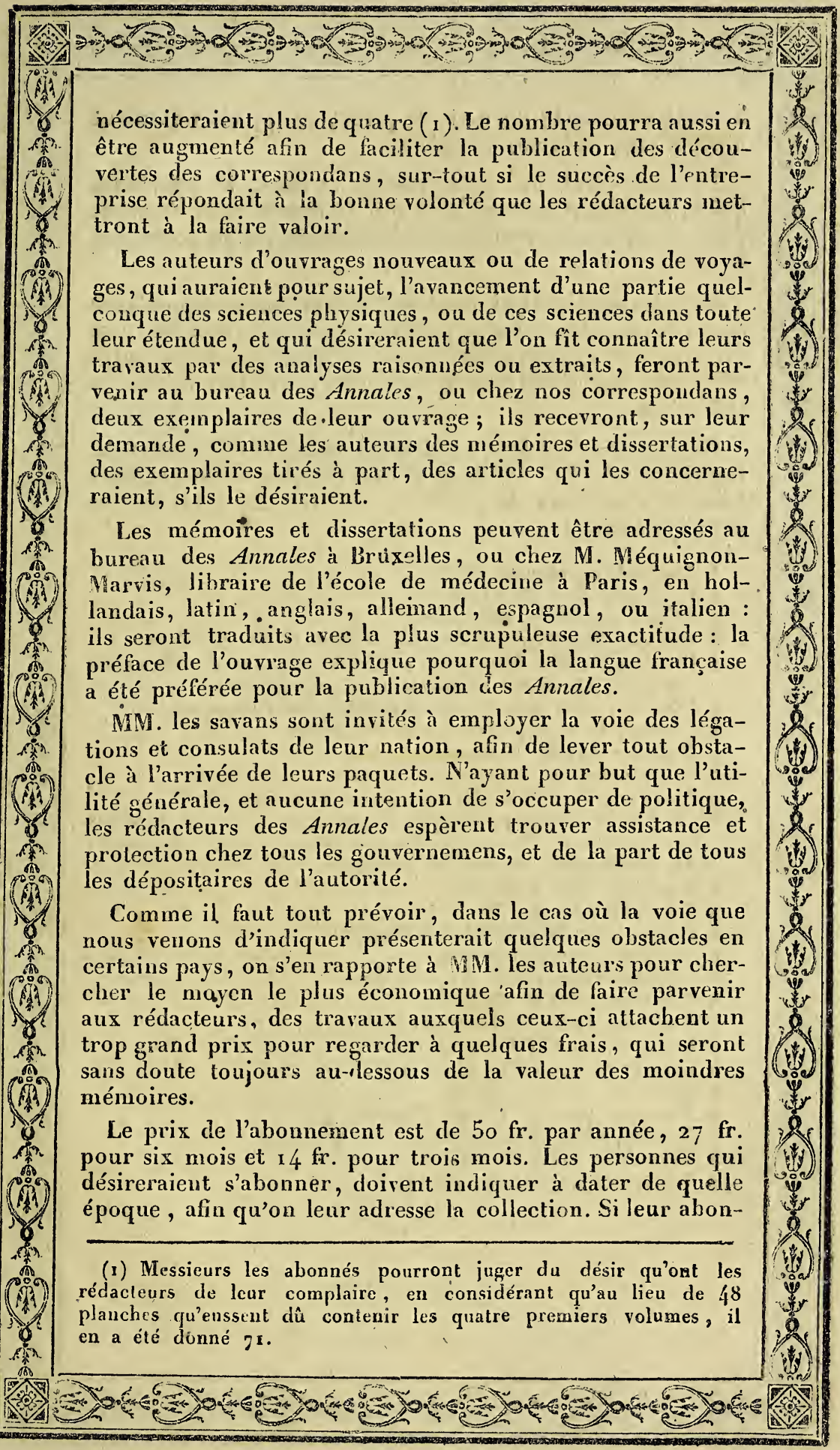




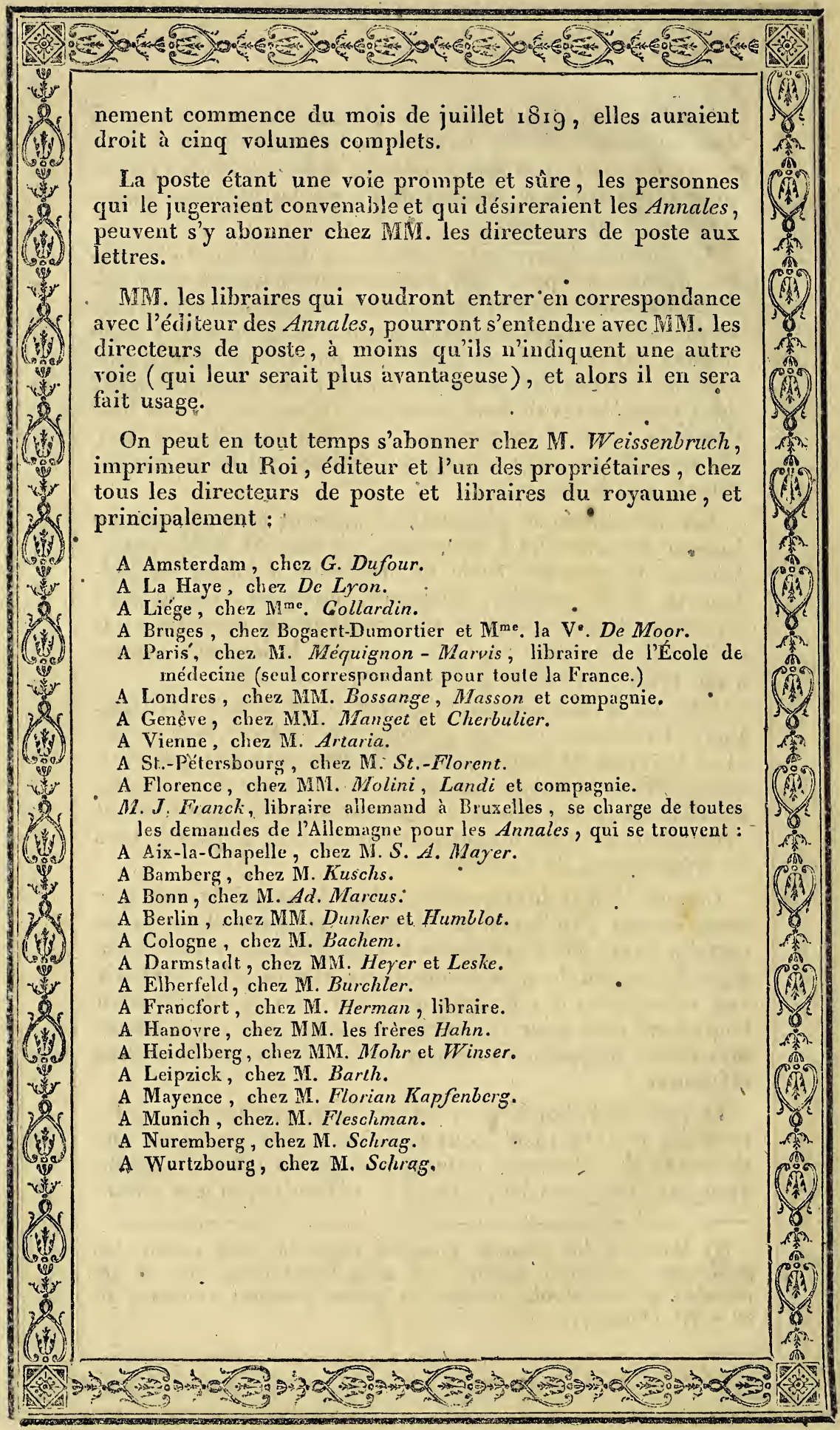




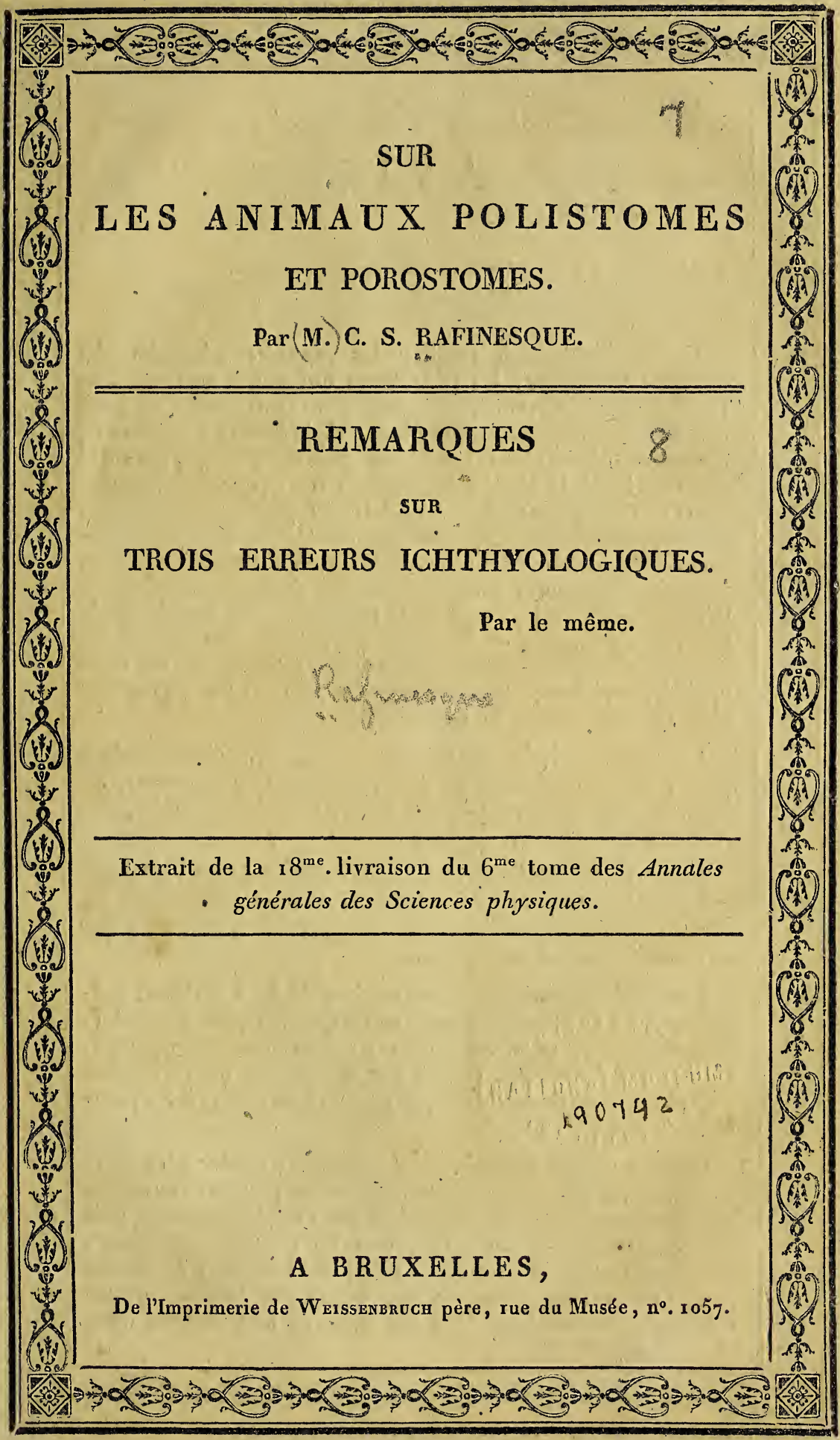




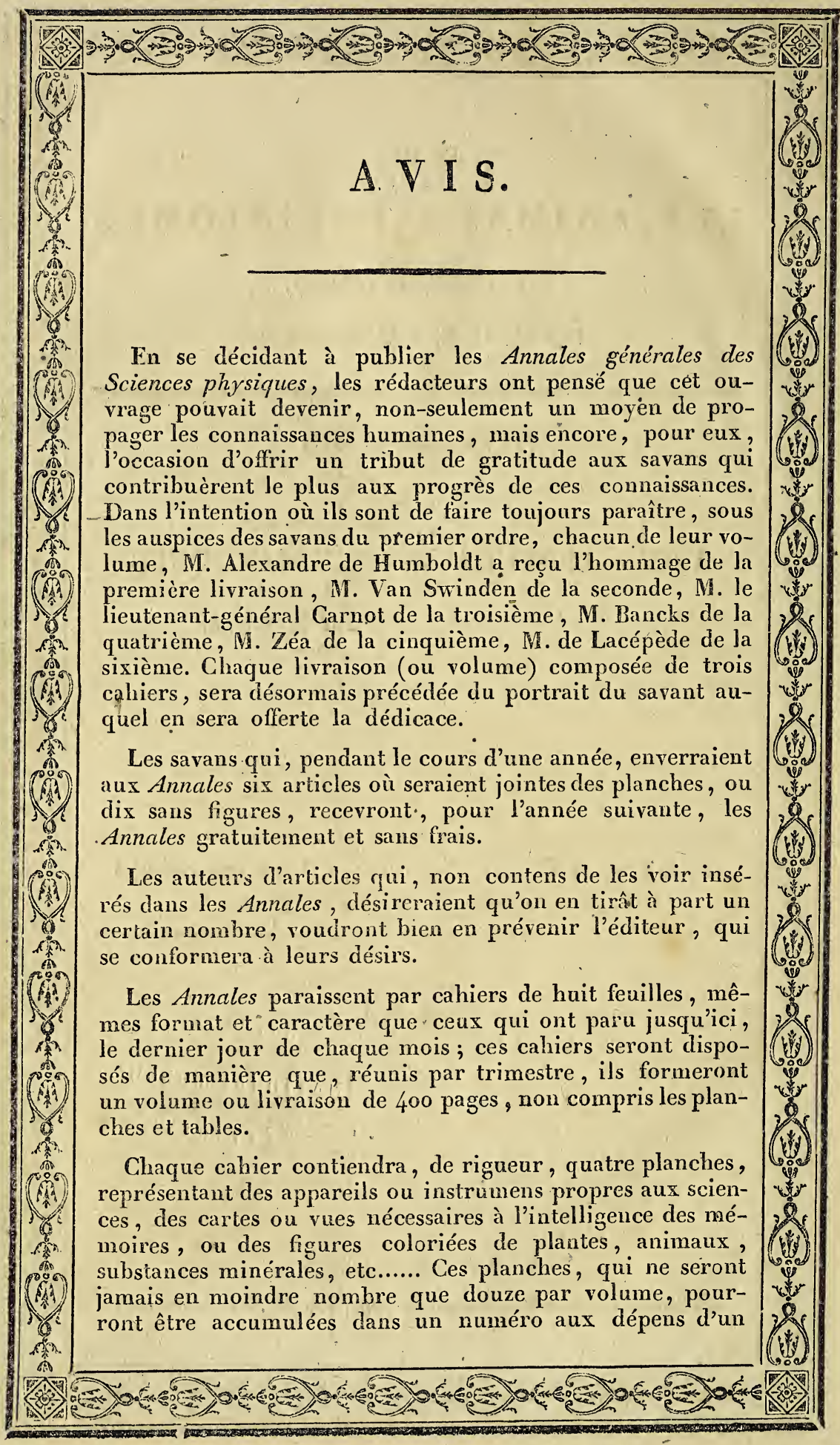




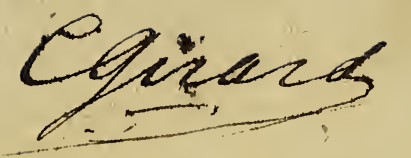

\section{SUR LES ANIMAUX POLISTOMES ET POROSTOMES.}

Par C. S. RAFINESQUE, professeur de botanique et d'histoire naturelle à l'ụniversité de Lexington en Kentucky.

Des erreurs accréditées par des savans illustres, admises tacitement comme des vérités démontrées par la foule des copistes et des esprits superficiels qui se contentent de croire sur parole, sont bien difficiles à détruire; néanmoins il est du devoir de ceux qui ont vérifié et constaté les faits réels qui les détruisent, de chercher à les divulguer et à éclairer le domaine des sciences.

11 semble, au premier coup d'oil, que c'est un paradoxe que d'admettre des animaux polistomes ou munis de plusieurs bouches, et encore plus des animaux porostomes ou sans houches apparentes, leurs pores en tenant lieu, comme dans les plantes; cependant rien n'est plus vrai que l'existence d'un grand nombre de ces êtres, parmi les Polypes, les Polypiers, les Méduses, etc.

Les polypiers ont été, pendant long-temps, le jouet des systèmes; on les a tantôt considérés comme des pierres, tantôt comme des plantes; mais les découvertes de Trembley, Donati, Ellis, etc. les fixèrent parmi les animaux, et l'on supposa qu'ils étaient construits par des polypes, ce qui était aussi vrai que de dire que les fenêtres construisent nos maisons. L'ingénieuse raillerie de Bernardin de St. Pierre, dans ses dialogues d'un voyageur, aurait dû. éclairer sur l'absurdité de cette fausse vue : car d'après lui les plantes seraient aussi des polypiers.

Mais Cavolini a prouvé depuis assez long-temps que les Polypiers, les Alcyons, etc. formaient des corps individuels, tout comme un arbre ou un cacte; qu'ils sont pourvus de plusieurs orifices ou bouches de diverses formes, propres à suppléer au défaut de locomobilité, etc. Cependant presque tous les naturalistes ont négligé ses vues sur ce fécond. sujet. 
Trantenant, l'on trouve encore dans tous les ouvrages modernes d'histoire naturelle, l'idée absurde des polypes constructeurs; l'on a tout au plus appliqué à quelques-uns d'entr'eux une nouvelle thẻorie, déduite de quelques faits particuliers, et qui est celle de l'agrégation ou consolidation de plusieurs individus ayant une prétendue existeace commune, ce qui après tout n'est que la vérité déguisée sous de faux termes.

C'est ce que nous voyons dans les savans ouvrages de MW. Cuvier, Lamark, Lamouroux, etc., et c'est ce que l'on répète dans tous les dictionnaires, encyclopédies, élémens, etc. Il est plus facile de copier des erreurs, que de rechercher la vérité, et quand elle est découverte, elle a souvent bien de la peine à percer les nuages de l'ignorance óu des préjugés scientifiques.

J'ai habité plus de vingt ảns les bords de la Méditerranée en Provence, en Italie et en Sicile : j'ai observé par moi-même, j'ai étudié avec attention tous les animaux marins, et les résultats de mes travaux m'ont convaincu que la théorie de Cavolini est la seule vraie, la seule admissible, et la seule qui démontre et explique les nombreux phénomènes que hous offrent les polypiers, etc.

Conduit par la voie de l'analogie et de l'évidence, j'ai reconnu que les animaux qui n'offrent aucun orifice extérieur, mais qui cependant agissent, se meuvent et croissent, doivent absorber leur nourriture sous une forme fluide ou gazeuse par des pores; et comme ces pores remplacent chez eux les bouches multiples et compliquées des polypiers, j'ai nommé ces animaux porostomes, et j'en ai formé la dernière classe animale, puisque c'est celle qui se rapproche le plus des plantes.

Les animaux polistomiques n'existent réellement que dans la neuvième ou avant-dernière classe animale; (Polypia) les Polypes. L'on ne trouve dans les classes supérieures des mollusques et des vers (Apalosia et Helmisia) que quelques apparences d'agrégation dans les Serpules, les

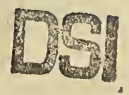


Anatifes, mais surtout les Salpaires, qui forment le chânon conduisant des mollusques aux polypes.

J'avais énoncé, dès 1814 , mes découvertes à cet égard, dans le Journal Encyclopédique de Sicile, et je lẹs avais détaillées dans moń Analyse de la Nature, publiée en 1815 , mais succinctement et synoptiquenent, de manière qu'elles ont échappé à l'attention des compilateurs, qui ne savent extraire des vérités que des ouvrages diffus, où le sujet est noyé dans les détails accessoires.

Je vais maintenant passer en revue les animaux qui offrent des bouches multiples, polypiformes ou poriformes, et j'espère.que ceux qui ont assez de bonne foi pour préférer la vérité pure et simple, à l'erreur couverte d'un voile brillant, voudront bien méditer les pensées que je leur pourrai suggérer et reconnaître l'évidence des faits.

Dans l'ordre Helminthia ou des vers parasites, presque tous les genres des familles Asthrenia ou Jenias, Fasciolatria ou Fasciolaires, Physelmia ou Hydatiens, et Scolexica ou Ascaridiens, ont plusieurs bouches évidentes, auxquelles l'on a donné le nom de suçoirs, parce qu'ils en ont souvent la forme. Cela est admis par les naturalistes, et tout orifice externe qui admet la nourriture est une bouche.

Le second ordre de Polypes, Fistulidia, n'offre que des genres monostomes; mais le suivant, Radiaria, a une famille entière polystomique : je l'ai nommée Polytremia; le type en a été le genre Rhizostoma de M. Cuvier, où il a reconna lui-même une multitude d'orifices ou bouches. J'ai réuni à cette famille toutes les Méduses qui ont plusieurs orifices, tels que les genres Cephea, Aurelia, Cassiopea, Ocyroe, Obelia, Eplyra, Euryales, etc., de Peron et Lesueur, outre plusieurs que j'ai découverts moi-même.

Le quatrième ordre parmi les Polypes, Monostomia, est caractérisé par une ouverture unique; mais le cinquième ou dernier, Polystomia, l'est an contraire par la multiplicité des orifices. Cet ordre est très-nombreux, car il 
renferme tous les Polypiers mols, flexibles on même pierreux. Je l'ai divisé en 5 familles et 12 sous-fanilles.

La première, Anaxia, offre des corps souvent fixes sans axe corné ni osseux; les uns sont ramifiés, tels que le genre Zoantha Lam. et mon genre Blophastoma, qui contient les vorticelles polistomes, tandis que les autres ont un corps simple, tels sont les genres Sinoicum de Phipps, Pyrosoma, (corps flottant) Per. et mes nouveaux genres Psadiroma, Polactoma, Chledripole, etc. Le genre Botryllus devra peut-être y être réuni.

La famille Axarchia, a un axe interne corné ou osseux, et le corps communément flottant, simple dans les genres Veretilla Lam., Funiculina Lam., etc., formant la sousfainille Veretillia, et rameux dans la sous-famille Encinia, contenant les genres Pennatula Lam. Encrinus Lam. Pentacrinus. Raf. Pentrema Raf., etc. Il n'y a acun genre qui offre plus évidemment l'organisation polystomique que le Pennatula, dont le corps flottant et mobile a un axe interne solide recouvert par une chair épaisse, où sont parsemées les ouvertures nutrifères.

J'ai nommé Dermopsia, la troisième famille contenant les Polypiers fixés à une axe pierreux recouvert par une peau corticiforme. La sous-famille Arthropsia a l'axe articulé; son type est le genre Isidia, tandis que les genres Corallium, Gorgonia, Antipathes, etc., forment le type de la deuxième sousfamille, où l'axe n'est pas articulé. Ici l'écorce animale et polystomique est encore très-évidente, les bouches sont munies de tentacules souvent floriformes, que l'on a depuis prises pour des polypes individuels quoiqu'elles ne jouissent pas d'une existence individuelle; elles ont seulement des mouvemens particuliers et indépendans, comme nos bras et nos jambes.

Les polypiers à corps pierreux sans peau corticiforme constituent la quatrième famille Lithopsia.C'est ici seulement que l'on pourrait mettre en doute l'existence polystomique mais ce doute n'est qu'apparent, car lorsqu'on examine ces 


\section{(85)}

les fait sécher à l'air ou à la fumée, et ensuite on les transà porte enfilés par un bout, avec un cordon. Lear grossear varie depuis six pouces de long jusqu'à deux pieds, et en général ils ne sont guères au-dessous d'un pied, ce qui est la grosseur du Muge lui-même, Les laites des mâles sont aussi salées quelquefois, quoiqu'on préfère les manger fraiches. On les prépare comme les oufs, dont ils ne diffèrent que par leur mollesse et leur blancheur. On lès nomme en italien Bottarga bianca ou Lattume. Chaque partie du. Thon se sale à part, en Sicile, etc. et obtient un nom particulier; le ventre que l'on nomme Sura ou Ventresea en italien, est la partie la plus estimée après la Boutarque. On ne néglige pas même la peau, les os, ni les intestins de ce poisson si utile; tout se sale, se mange et trouve son prix. 







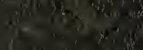

$\frac{2}{25}$

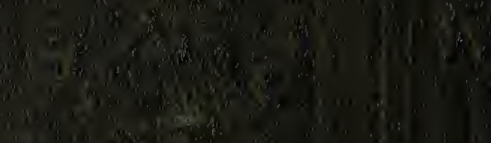

$$
\begin{aligned}
& \text { (a) }
\end{aligned}
$$

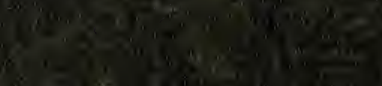

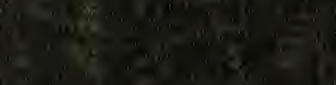

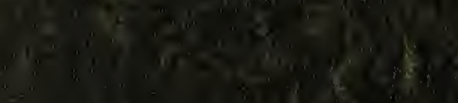

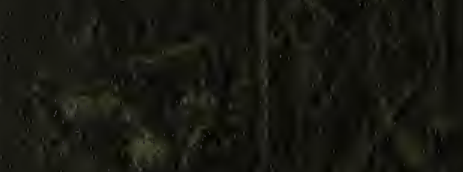

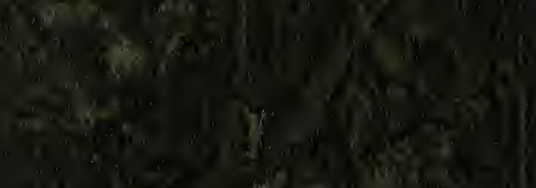

$$
\begin{aligned}
& \text { No }
\end{aligned}
$$

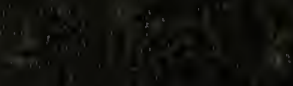

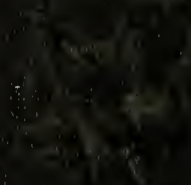

$$
\begin{aligned}
& \operatorname{anc}=1
\end{aligned}
$$

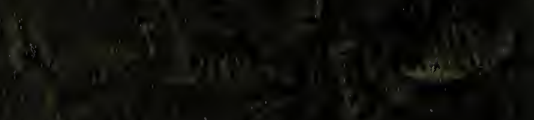

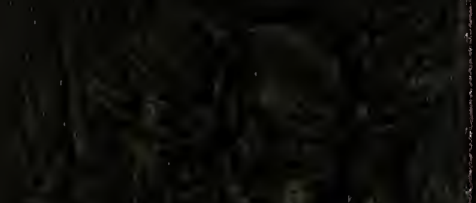

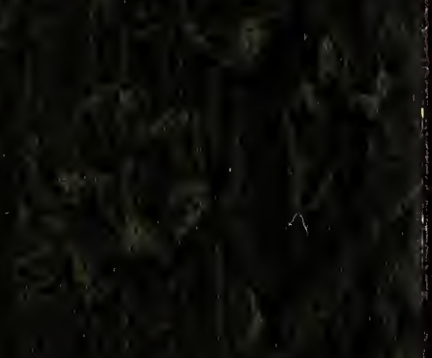

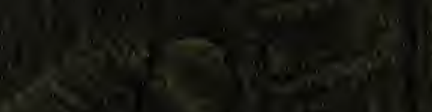

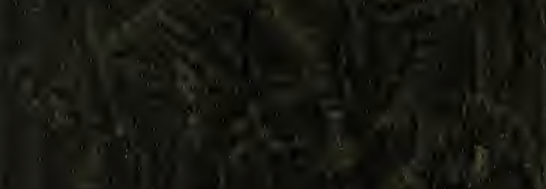

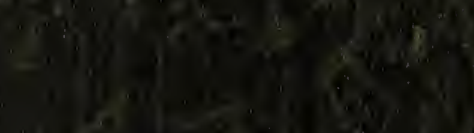

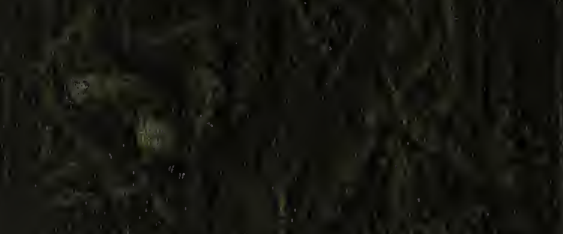

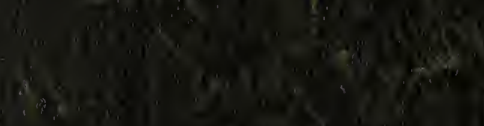

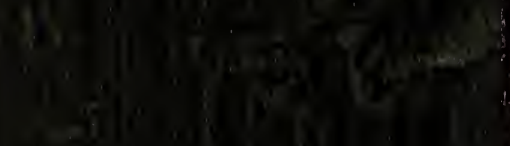

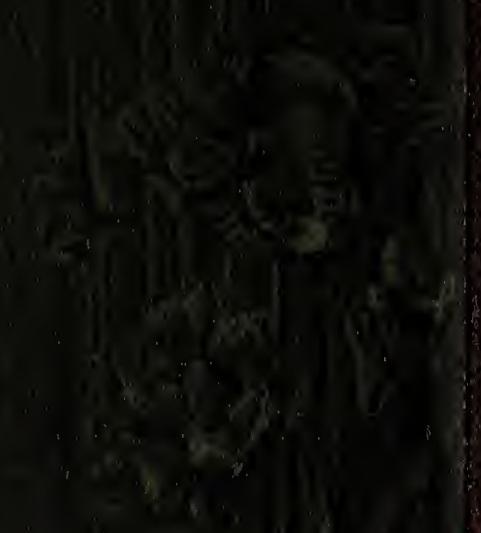

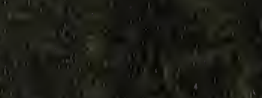

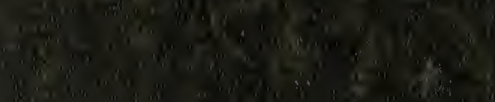

